\title{
Melanoma of the Oral Cavity: About Two Cases and Review of Literature
}

\author{
F. Elomrani ${ }^{1 *}$, H. Mouzount ${ }^{1}$, I. Ouziane ${ }^{1}$, R. Khmamouch ${ }^{1}$, S. lkhoyali ${ }^{1}$, A. Boukir ${ }^{1}$, M. ElKabous ${ }^{1}$, \\ S. Boutayeb ${ }^{1}$, H. Mrabti ${ }^{1}$, B. Elkhannoussi ${ }^{2}$, H. Errihani ${ }^{1}$ \\ ${ }^{1}$ Department of Medical Oncology, National Institute of Oncology, Rabat, Morocco; ${ }^{2}$ Department of pathology, National Institute of \\ Oncology, Rabat, Morocco. \\ Email: *elfadwa512@hotmail.fr
}

Received January $8^{\text {th }}, 2013$; revised February $27^{\text {th }}, 2013$; accepted April $10^{\text {th }}, 2013$

Copyright (C) 2013 F. Elomrani et al. This is an open access article distributed under the Creative Commons Attribution License, which permits unrestricted use, distribution, and reproduction in any medium, provided the original work is properly cited.

\begin{abstract}
Mucosal malignant melanoma of the oral cavity is an extremely rare condition. It has a poor prognosis. Here we report two typical cases of malignant melanoma. In the first case, 64 years old man developed an exophytic tumor in the hard palate. Head and neck and chest computerized tomography scan showed a large aggressive tumor of the hard palate. The patient also had multiple lung metastases and a cervical lymph node. The second case is a 73 years old woman presenting a burgeoning mass on the right palate. Facial CT reveals a malignant tumor lateralized in the right palate with ipsilateral metastatic lymph nodes. Histological with immunohistochemical studies assigned both cases to a malignant melanoma. Due to the rarity of oral malignant melanomas, case reports are a necessary source of information.
\end{abstract}

Keywords: Melanoma; Oral Cavity

\section{Introduction}

Melanoma of the oral cavity (MOC) is a rare malignant disease, which present only $2 \%$ to $8 \%$ of all melanomas [1]. MOC is often discovered in an advanced stage, they had a poor prognosis.

The most common locations are the palate and maxillary gingiva. Metastatic melanoma most frequently affects the mandible, tongue, and buccal mucosa [1]. The relative rarity of mucosal melanomas has dictated that tumor staging should be based on the broader experience with cutaneous melanoma. Oral melanomas seem uniformly more aggressive; they spread and metastasize more rapidly than other oral cancers or cutaneous melanomas [1]. Early recognition and treatment greatly improves the prognosis. Unfortunately, oral mucosal melanomas have by far the worst prognosis and an average of 5 years survival [2]. Here we report 2 cases of oral malignant melanoma.

\subsection{Case Presentation 1}

A 64 years old Moroccan male patient without medical history, operated one year ago for an extensive ulceration

\footnotetext{
"Corresponding author.
}

in the palate. Histological analysis confirmed an Oral Melanoma of the Palate with positive HMB45 and Melan A. Recently the patient developed ulceration in the operated area (Figure 1). A Biopsy was performed and pointed out a malignant melanoma. Physical examination revealed an exophytic tumor on the left palate, and cervical lymph node measuring approximately $4 \mathrm{~cm}$. A computerized tomography (CT) of head, neck and chest shows a large aggressive tumor of the hard palate lateralized to the left. The tumor is associated with cervical lymph node greater than $30 \mathrm{~mm}$ in diameter. There were multiple lung metastases, but no brain metastases could be detected. The multidisciplinary committee decided a treatment by first line chemotherapy based on Dacarbazine $750 \mathrm{mg} / \mathrm{m}^{2}$ on day 1 every 28 days.

\subsection{Case Presentation 2}

73 years old Moroccan female patient without medical history presented since 5 months a burgeoning mass on the right palate gradually increasing volume. Clinical examination found a burgeoning mass ulcerated taking the whole palate (Figure 2), and right cervical lymph node measuring $2 \mathrm{~cm}$ of diameter.

Facial CT revealed malignant tumor lateralized in the 


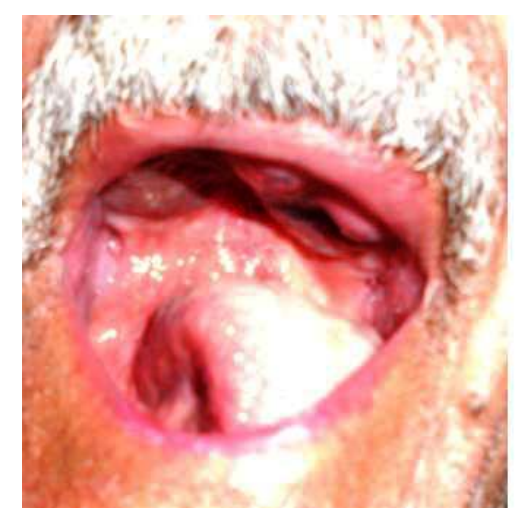

Figure 1. Extensive ulceration and brown irregular macula of the hard Palate.

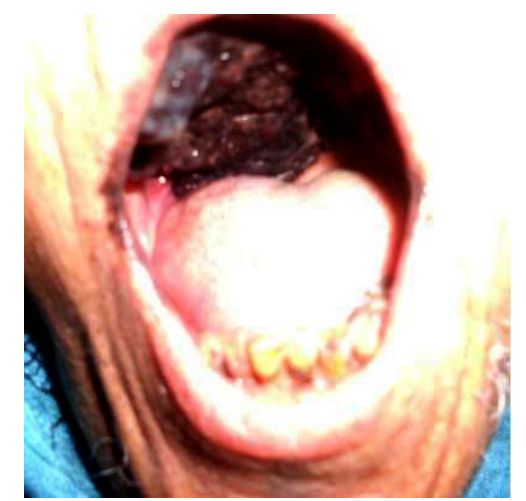

Figure 2. A brown patch that extended to cover the hard palate and involved the alveolar edge region.

right palate with ipsilateral metastatic lymph nodes.

A biopsy of the process was performed and showed squamous mucosa infiltrating with an ulcerative undifferentiated malignant proliferation (Figure 3(a)). It made diffuse sheets of fusiform cells with elongated nuclei and inconspicuous cytoplasm containing a brownish pigment of melanin. The nuclei show moderate cytonuclear atypia with numerous atypical mitotic (Figure 3(b)). Immunohistochemical study showed positive staining for HMB45 and Melan A and negative for cytokeratins (Figure 4).

The multidisciplinary committee decided to treat the patient with chemotherapy based on Dacarbazine 750 $\mathrm{mg} / \mathrm{m}^{2}$ on day 1 every 28 day.

\section{Discussion}

The incidence of mucosal melanoma is lower than in the cutaneous ones. Jackson and Simpson have indicated that malignant melanoma of the oral cavity represents less than $2 \%$ of all melanomas [1], and Reddy et al. have indicated an incidence of $0.4 \%-1.3 \%$ [2]. Primary oral melanomas are extremely rare in the USA and account less than $2 \%$ of all melanomas. At 1.2 cases per 10 million people per year, the annual incidence of oral melanoma is very low [2]. Recent and major investigations in

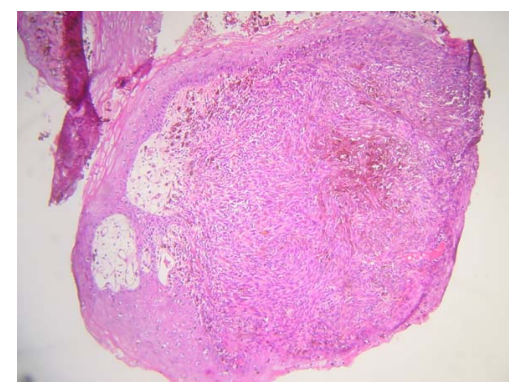

(a)

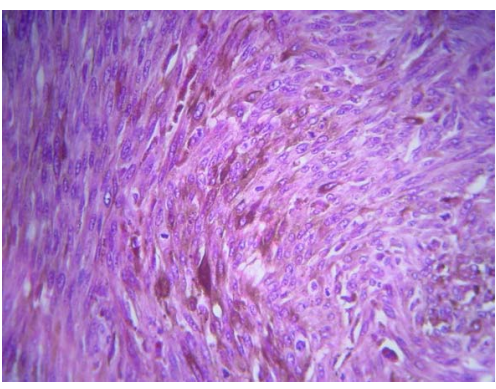

(b)

Figure 3. (a) Microphotograph showing a squamous mucosa infiltrating with an ulcerative undifferentiated malignant proliferation (hematoxylin-eosin magnification $\times 4$ ); (b) Microphotograph showing diffuse sheets of fusiform cells with elongated nuclei and incouspious cytoplasm containning pigment of melanin (hematoxylin-eosin magnification $\times 40$ ).

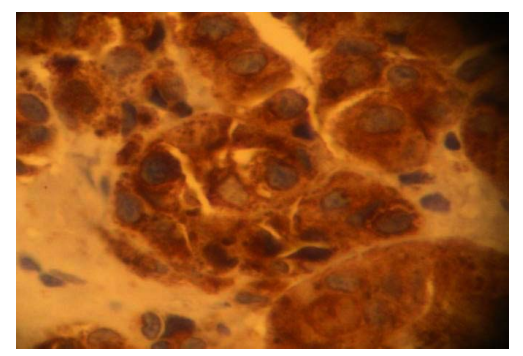

Figure 4. Microphotograph showing positive staining of tumor cells for HMB45 (Avidin Biotin at magnification $\times 40)$.

Africa showed oropharyngeal melanoma as $1.7 \%$ of all melanomas, Van der Wall et al. showed that only $2.5 \%$ of all melanomas were oral primaries [3]. This malignancy is rarely identified in patients under the age of 20 years; the highest incidence was reported in the fifth decade. Males appear to be affected more often than females and whites more than blacks [1]. For our cases, the two patients had more than 65 years old. According to Tanaka et al., oral melanomas could be classified into five types based on their clinical appearance: pigmented nodular, non pigmented nodular, pigmented macular, pigmented mixed and none pigmented mixed. The clinical coloration has a wide range, which can appear as black, grey, purple and reddish. The tumors are asymmetric, irregular in outline and occasionally multiple [4]. 
Pain, ulceration and bleeding are rare in oral melanoma until late in the disease [5]. In our cases the tumors are irregular, asymmetric, ulcerated and their color was brown. Most malignant melanomas arises de novo; some may arise from pre-existing nevi, especially atypical (dysplastic) nevi, or congenital hairy nevi [6]. Many genes are implicated in the development of melanoma, including CDKN2A (p16), CDK4 (chromosome 12q15), RB1, CDKN2A (p19) and PTEN/MMAC1 $[7,8]$. The etiology of oral melanoma is unknown. Exposure to sunlight, denture irritation, chewing tobacco with betel nut and smoking has been implicated as etiologic factors in the past. However, there has been no evidence to support these theories. Currently; most oral melanomas are thought to arise de novo [9]. Few symptoms are found early in the malignancy. The patient's attention may be drawn to the lesion by the presence of a swelling mass, especially in a pigmented area, possible interference with the fit of dentures, hemorrhage, and loosening of teeth [10,11]. Pain is an uncommon symptom of malignant melanoma, generally found in the advanced stages [12]. The tumor causes extensive destruction of the underlying bone in $78 \%$ of cases $[13,14]$.

Differential diagnosis for oral melanoma includes oral melanotic macule, smoking-associated melanosis, melanoplakia, pituitary-based Cushing's syndrome, post inflammatory pigmentation, melanoacanthoma, melanocytic nevi of the oral mucosa, blue nevi, spitz nevi, Addison's disease, Peutz-Jeghers syndrome, amalgam tattoo, Kaposis's sarcoma, physiologic pigmentation and many other conditions sharing macroscopic characteristics with oral melanoma [15]. This neoplasm should also be differentiated histopathologically from other malignant entities, such as poorly differentiated carcinoma and large cell anaplastic lymphoma [15]. Most suspected melanomas are diagnosed with a sensitive marker: S100. More specific markers such as Melan-A, tyrosinase or HMB45 can confirm the diagnosis. As for the other markers, Ki-67 is commonly used as an adjunct in distinguishing benign nevi from melanoma. The search for a specific marker for these lesions must continue [16]. Some of the more recently studied proliferation markers include HDM2 and the Growth arrest DNA damage (GADD) proteins to show that they may have real promise as prognostic markers [16].

Surgery is the mainstay of treatment, but can be difficult due to anatomic restraints. Although melanoma is classically not radiosensitive, occasional patients have shown good response to radiation therapy, especially in early or in situ melanomas [4]. Immunotherapy has been successfully used but chemotherapy has demonstrated a relatively low response rate. Dacarbazine, INF-gamma and INF-alpha-2b have been described as chemotheraupetical and immunotherapeutical treatments associated with Bacillus-Calmette-Guerin vaccine and recombinant interleukin-2 in different combinations [4,17]. Clinical trials of Ipilimumab, a human monoclonal antibody, have shown encouraging results in therapy for metastatic melanoma. The drug blocks regulation of cytotoxic T-lymphocytes and allows sustained immunologic activity against melanoma and other malignancies. Ipilimumab is now approved by the US Food and Drug Administration (FDA) for unresectable or metastatic melanoma [17]. Vemurafenib is a specific inhibitor of signaling by mutated BRAF is recommended for patients with V600E mutation of BRAF gene for untreated metastatic melanoma [18].

Experience with oral malignant melanoma is largely derived from single cases. Anecdotal reports describe success with IFN-alfa or hyperfractionated radiation therapy [17]. Many cancer centers follow surgical excision with a course of IL-2 as adjunctive therapy to prevent or limit recurrence. Peginterferon alfa-2b (Sylatron) has been approved by the FDA for melanoma with microscopic or gross nodal involvement within 84 days of definitive surgical resection including complete lymphadenectomy [17].

Because of the rarity of the lesions, assembling a cohort study group to evaluate the different therapeutic regimens is difficult. The hope is that future research will incorporate standardized multimodal therapy, such as those used in the treatment of cutaneous melanoma [19].

Most oral melanomas are large at presentation and have poorer prognosis than cutaneous melanoma. In general, the survival rates are poor and are worse for those with metastasis [12]. Chaudhry et al. reported that the average duration of life from the point of diagnosis was about 18 months. Sampat and Sirsates reported that 79\% of patients died within 5 years. In addition, Vairaktaris et $a l$. showed that the 5-year survival rate of intraoral melanoma does not exceed 5\% - 9\% [19].

\section{Conclusion}

Primary oral mucosal melanomas are exceedingly rare and biologically aggressive malignancies. Oral melanoma clinically mimics many other pigmented lesions of the oral cavity. It is essential to include oral examination in full body skin examinations, dentures should be removed for examination. Suspicious pigmented and nonpigmented lesions should be biopsied appropriately. Because early diagnosis and intervention results in better prognosis.

\section{REFERENCES}

[1] D. Jackson and H. E. Simpson, "Primary Malignant Melanoma of the Oral Cavity," Oral Surgery, Oral Medicine, Oral Pathology, Vol. 39, No. 6, 1975, pp. 553-559. doi:10.1016/0030-4220(75)90194-2 
[2] N. J. D’Silva, Z. Kurago, P. J. Polverini, C. T. Hanks and A. F. Paulino, "Malignant Melanoma of the Oral Mucosa in a 17-Year-Old Adolescent Girl,” Archives of Pathology \& Laboratory Medicine, Vol. 126, No. 9, 2002, pp. 11101113.

[3] R. I. van der Waal, G. B. Snow, A. B. Karim and I. Van der Waal, "Primary Malignant Melanoma of the Oral Cavity: A Review of Eight Cases," British Dental Journal, Vol. 176, No. 5, 1994, pp. 185-188. doi:10.1038/sj.bdj.4808406

[4] M. Hashemipour, "Malignant Melanoma of the Oral Cavity,” Journal of Dentistry, Vol. 4, No. 1, 2007, pp. 44-51.

[5] B. Colllins, E. Leon Barnes Jr. and J. Abernethy, "Oral Malignant Melanoma,” Journal of Clinical Oncology, Vol. 55, 2005, pp. 74-108.

[6] Bork, Hoede, Korting, Burgdorf and Young, "Diseases of the Oral Mucosa and the Lips,” W. B. Saunders, Philadelphia, 1996, pp. 336-340.

[7] R. Rajendran and B. Sivapada Sundaram, "Benign and Malignant Tumors of the Oral Cavity,” In: Shafer, Hine and Lavy, Eds., Shafer's Text Book of Oral Pathology, Elsevier India, 2009, pp. 120-127.

[8] M. Singh, J. Lin, T. L. Hocker and H. Tsao, "Genetics of Melanoma Tumerogenesis,” British Journal of Dermatology, Vol. 158, No. 1, 2008, pp. 15-21. doi:10.1111/j.1365-2133.2007.08316.X

[9] A. D. Rapidis, C. Apostolidis, G. Vilos and S. Valsamis, "Primary Malignant Melanoma of the Oral Mucosa," Journal of Oral and Maxillofacial Surgery, Vol. 61, No. 10, 2003, pp. 1132-1139. doi:10.1016/S0278-2391(03)00670-0

[10] N. E. Steidler, P. C. Reade and B. G. Radden, "Malignant Melanoma of the Oral Mucosa," Journal of Oral and Maxillofacial Surgery, Vol. 42, No. 5, 1984, pp. 333-336. doi:10.1016/0278-2391(84)90116-2

[11] S. R. Prabhu, D. F. Wilson and D. K. Daftary, "Oral Dis- eases in the Tropics,” Oxford University Press, New York, 1992, pp. 460-461.

[12] D. Jackson and H. E. Simpson, "Primary Malignant Melanoma of the Oral Cavity,” Oral Surgery, Oral Medicine, Oral Pathology, Vol. 39, 1975, pp. 553-559. doi:10.1016/0030-4220(75)90194-2

[13] N. K. Wood and P. W. Goaz, "Differential Diagnosis of Oral and Maxillofacial Lesions,” 5th Edition, Maryland Heights, 1997, pp. 67-68, 190.

[14] B. F. Barker, W. M. Carpenter, T. E. Daniels, M. A. Kahn, A. S. Leider, F. Lozada-Nur, et al., "Oral Mucasal Melanomas: The WESTOP Banff Workshop Preceedings: Western Society of Teachers of Oral Pathology,” Oral Surgery, Oral Medicine, Oral Pathology, Oral Radiology and Endodontics, Vol. 83, No. 6, 1977, pp. 672-679. doi:10.1016/S1079-2104(97)90318-8

[15] M. S. Hashemi Pour, "Malignant Melanoma of the Oral Cavity: A Review of Literature,” Indian Journal of Dental Research, Vol. 19, No. 1, 2008, pp. 47-51. doi:10.4103/0970-9290.38932

[16] S. J. Ohsie and G. Peter, "Immunohistochemical Characteristics of Melanoma," Journal of Cutaneous Pathology, Vol. 35, No. 5, 2008, pp. 433-444. doi:10.1111/j.1600-0560.2007.00891.x

[17] B. Collins II, “Oral Malignant Melanoma,” Emedecine Medscape, 2011.

[18] P. B. Chapman, A. Hauschild, C. Robert, et al., "Improved Survival with Vemurafenib in Melanoma with BRAF V600E Mutation," The New England Journal of Medicine, Vol. 364, No. 26, 2011, pp. 2507-2516. doi:10.1056/NEJMoa1103782

[19] E. Vairaktaris, I. Iatrou, V. Ragos, K. Petraki and C. Martis, "Primary Melanoma of the Oral Cavity," Helleniko Periodiko gia Stomatike \& Gnathoprosopike Cheirourgike, Vol. 4, 1989, pp. 31-36.

\section{List of Abbreviations Used}

MOC: Melanoma of the oral cavity

CT: Computed tomography

GADD: Growth arrest DNA damage

FDA: Food and Drug Administration 